\title{
MANAGEMENT OF VIRTUAL ENTERPRISES: \\ A CASE STUDY OF GERMAN RAPID PROTOTYPING SMES
}

\begin{abstract}
Frank Wagner
Head of Competence Center R\&D Management,

Fraunhofer Institute for Industrial Engineering (Fraunhofer IAO),

Institute for Human Factors and Technology Management (IAT, Universität Stuttgart),

Frank.Wagner@iao.fraunhofer.de
\end{abstract}

Jochen Eichert

Fraunhofer Institute for Industrial Engineering (Fraunhofer IAO),

Institute for Human Factors and Technology Management (IAT, Universität Stuttgart),

Jochen.Eichert@iao.fraunhofer.de

Roman Schirra

Fraunhofer Institute for Industrial Engineering (Fraunhofer IAO), Institute for Human Factors and Technology Management (IAT, Universität Stuttgart),

Roman.Schirra@iao.fraunhofer.de

GERMANY

This paper describes a case study of the results extracted from the research done in recent EU-projects on current and best practices for maintaining and optimizing Virtual Enterprises (VE). It is based on the example of a Virtual Enterprise practiced by German small and medium enterprises (SME) operating in the field of rapid prototyping (RP) and modelling within the automotive sector.

\section{INTRODUCTION}

To stay competitive, especially in comparison to larger companies, small and medium enterprises (SME) nowadays are more and more in the need of establishing Virtual Enterprises with other small companies in order to be able to virtually cover an extended field of business activities and thus having a better (stronger) position at the markets. At the same time, it is also necessary to fully integrate the customers, suppliers and cooperating partners/companies, that are involved in such an overall project, as far as possible. The necessity of collaborating even gains in importance when the developed and manufactured products and related services are getting more complex.

The Virtual Enterprise (VE) that is described in this paper consists of four German SMEs which are all operating in the field of rapid prototyping (RP) and 
modelling within the automotive sector. This case study describes how the cooperating partners of this $\gg$ Rapid Development VE« cope with these modern challenges, respectively how they are proceeding on their way of optimizing their workflows and business processes as well as enhancing their supporting information technology (IT)-infrastructure.

\section{THE RAPID DEVELOPMENT VIRTUAL ENTERPRISE}

The companies within this Virtual Enterprise are successful in the business of rapid prototyping, mainly for the automotive industry. They all use the latest technologies to create prototypes and models in the highest possible quality and are able to deliver their products in best time. Thus these companies are far ahead of their competitors at the German market. To keep this advantage of being among the fastest in the field of prototyping, they depend on the most reliable and advanced technologies that are available.

The four SMEs (Cirp, Hofmann, Kurz and Leotech) that form this Virtual Enterprise are shortly introduced in the following paragraphs.

Cirp Informationssysteme und Rapid Prototyping GmbH (www.cirp.de) was founded as a rapid prototyping service bureau. In the meantime, Cirp has evolved into a system provider who offers holistic solutions to its customers from design and product development up to the complete production of small series. Besides that, Cirp also has its own products for the advertising industry. The company has twelve employees. In addition, Cirp offers the following RP methods and technologies to its customers: selective laser sintering (SLS), stereo-lithography (SLA), die-casting, vacuum moulding and rapid tooling. In short, the core competences that Cirp is offering are innovative solutions with high quality prototypes delivered in the shortest time.

Josef Hofmann Modellbau + Vorrichtungsbau (www.hofmod.de) was founded as a company with two employees, which specialised in casting patterns and interior finishing. By now, the company has sixty-five employees. Further competencies are computer aided design (CAD), rapid prototyping with vacuum castings, five-axis numerically controlled (NC) milling and drilling, automotive light technology (with main focus on design, functionality and display models) and fused deposition modelling (FDM) technology. Moreover Hofmann is involved in the production of "test cubing", which represents a complete car body, where the models (generally made out of aluminium) are built completely from design data. The company is by now also certified according to DIN ISO 9001.

Modellbau Ralph Kurz (www.KurzModellbau.de) is a small engineering company currently with sixteen employees and closely cooperating with the other VE-partners. The main business fields of Kurz are classic model making and the follow-up technology vacuum moulding for small series of parts and modules for the automotive and machine industry. In the meantime, Kurz is, by its own account, with seven vacuum moulding machines the biggest company in Europe using this technology for rapid prototyping.

Leotech Rapid Prototyping und Werkzeugbau GmbH (www.leotech.de) is a very young but innovative service bureau in the field of metal prototypes for rapid tooling and mould-making and by now has four employees. It offers metal-casted prototypes 
within a few days (normally the processing time averages out at a few weeks) and also produces cast tools for injection moulding. Leotech can rely on the ITinfrastructure and engineering know-how mainly of its partner Cirp, which offers the required complementary services. Big enterprises appreciate the consultancy of Leotech and prefer the company as a supplier especially for time critical parts.

\section{THE PRINCIPLE OF COOPERATION}

The way of cooperation between the partners is quite special, because each partner of this VE offers the entire network performance to potential customers. Therefore the VE as a whole can offer the complete range of available competencies and services of all the companies together. This procedure also results in a better standing when it comes to the negotiation of contracts with bigger companies. In case, that a competency or service is available at more than one partner of the VE, each partner is also a direct competitor of the others.

The four German companies have mainly two reasons for building their Virtual Enterprise: technology sharing and capacity sharing. Technology sharing means that if a potential customer requests a technology or prototyping method which is not directly available at this contacted company, the request can still be processed if this service is offered by one of the VE-partners. On the other hand capacity sharing is meant in the sense that some of the partners offer the same services, but the company with the order from a customer has no free capacity and therefore hands over this order to the VE-partner, who is able to supply the needed resources. The benefit for all partners is that peaks of utilization can be avoided and thus a stable utilization rate for all partners can be achieved.

Since the partners are mainly working within the automotive sector (i.e. $70 \%$ of an average of ten to twenty simultaneously running projects with a duration between one day and six weeks), the required transparency of this branch directly reveals the major challenge for this networking Virtual Enterprise. Additional challenges are the documentation of tasks and projects (including quality, traceability and version control) as well as the coordination of the VE-members and the overall management of new and running projects. Thus the core competencies, sales strategies and corresponding information have to be presented at a very high level. In this context, it is not sufficient to just provide a transparent view of the complex interorganisational workflow, moreover it is essential, that all relevant process information and data have to be fully synchronised with all involved partners. 


\section{NEED FOR ACTION / IMPROVEMENTS}

Based on the insights of the previous chapter especially with respect to the future competitiveness, the four companies have realised their need for action and thus are all participating in various EU-projects related to the research on optimising and improving Virtual Enterprises.

\subsection{Requirements engineering}

As a first step, the VE respectively the four companies have been analysed in the requirements engineering phase under consideration of the following main topics:

- Organisation,

- Processes and

- IT-infrastructure.

\section{Organisation}

In contrast to most of their competitors, the German VE has no legal basis, contracts or fixed organisational (e.g. no common sales structures) or technological connections. The partners are actually competitors in their business and it is their free decision with whom they want to cooperate in a certain project. The allocation of all available resources is done independently, meaning that each partner is responsible only for his own planning. In addition the technological processes are independent from profit sharing. As mentioned before, the cooperation model between the companies is mainly focused on resource sharing (technology and capacity).

The culture of the cooperation is primarily based on confidence and trust. In other words, the partners know each other already for a long time and are able to predict very well how the others will behave. This predictability is the key principle of the VE. The executives of each partner build this confidence and trust, the project managers are planning and controlling the projects and the officials in charge (workers) stay in direct contact to their counterparts at the partner companies. The workers have all the freedom that is necessary to adjust the actual processes and adapt to possible changes. Since all departments of Cirp, Kurz and Leotech as well as the entire rapid prototyping department of Hofmann are involved in the VE, the employees know each other and this circumstance eases the cooperation. The coordination up to now is mainly done by acclamation via telephone, fax or email. Still one of the biggest organisational problems of each partner is to hire highly motivated employees who are willing to work in a flexible but unpredictable environment.

\section{Processes}

The most critical processes of the VE as well as for each partner are project planning, project controlling, order processing and order fulfilment. Typical is that a customer has one direct contact person at exactly one single partner. The customer usually does not know anything about the existence of the VE. All that counts for the customer is the requested service. The partner in charge (in accordance with the customer) has full control over the project and is absolutely free to decide whether to contact additional companies or not. Special types of models and files are created by 
every involved partner, assembled, and then exchanged between the partners, thereby benefiting from resource sharing. An online tracking system for the customers and an intra-organisational resource planning system would be a helpful supporting system. Until now, no project management software or other IT-systems are used for managing the VE processes. The project tasks are defined by milestones and observed without any IT-support, a short-term conflict management is working on human basis.

Offers can be calculated directly, if the know-how for the order fulfilment (for all tasks including those of the partners) is available in the company, which has been contacted by the customer. Otherwise, arrangements have to be made with the partners but then no extensive negotiations are necessary, because most of the costs are known by experience. This experience usually is much more accurate and effective than the use of existing calculation tools. Oral orders are made at first and then always followed by written orders for project planning and documentation. During the workflow, the biggest waste of time occurs for simple tasks like information research for materials, tolerances and measures, the transportation of parts between the partners and because of the lack of the availability of partners to make arrangements or to get feedback on important questions. Other disadvantages in the workflow are the general lack of project documentation, the decrease of documentation received from customers and sometimes, as already mentioned above, coordination problems.

\section{IT-Infrastructure}

All the partner companies use common data formats, the Internet, FTP-servers, Odette or email to exchange their project files. Contact to the customers and coordination between the partners is done mainly by telephone, fax or email. For resource planning, time scheduling and the definition of deadlines the partners internally use Microsoft Outlook. Most of the project information is stored on paper created with common office applications.

Responsible for the management of the project data is always the partner who has the direct contact to the customer. However, there are no special IT-systems used for coordination, planning and communication between the partners. The production planning and controlling is done manually. The only IT-systems in use are general financial business solutions.

Hence a common project management system with workflow, version control and resource planning would substantially improve the workflow of the VE as a whole. The use of a common web-based information platform could be used as a material database (basic properties of used materials in different processes) and for sharing the offer files. With systems like these, approximately $90 \%$ (a rough estimation by the VE-partners) of the coordination problems could be solved and the process speed increased. To further enhance the transparency of the production processes internally and towards the customer, an online tracking system would be very useful. 


\subsection{Workflow optimisation and IT selection}

After this phase of requirements engineering, it was obvious, that, even though this "Rapid Development VE« is operating very successful at the market, there are still enough fields which need improvement. Therefore the succeeding task was the optimisation of the workflow, which covers the entire product development cycle ranging from the customer request, the definition of an offer, the order up to the product creation and delivery, including order tracking and project management. The figure below shows a simplified model of this workflow as well as the interconnections inside the VE.

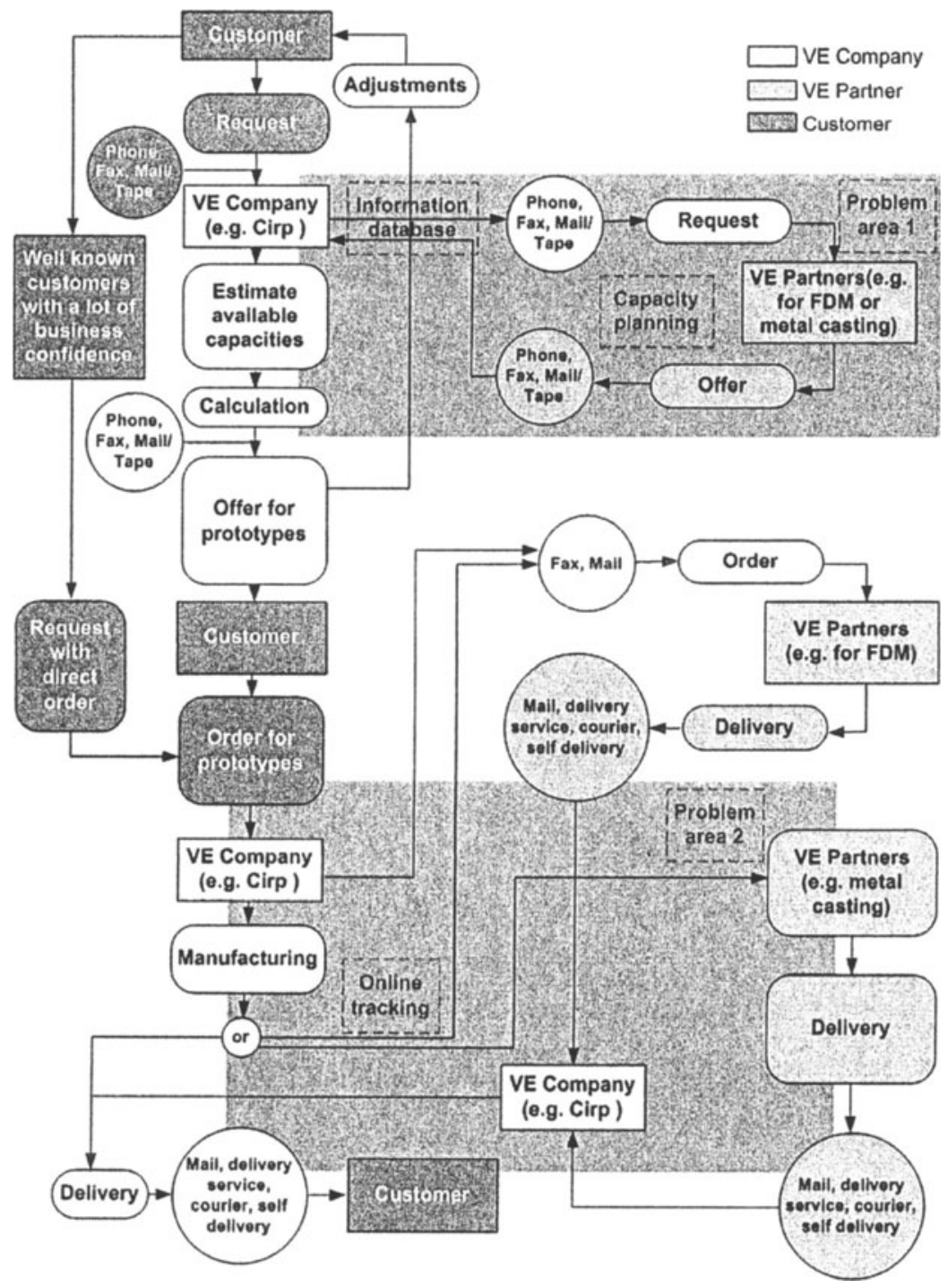

Figure 1 - Workflow of the »Rapid Development VE« 
For this VE the main improvements could be achieved by an optimisation against the background of an integrated IT-support for all the process steps, since the procedure of the workflow as a whole has proven its general usefulness in daily business. According to this revised workflow, the selection of an appropriate ITsystem was carried out.

The best technical solution for the VE, with full IT-support of all phases of the workflow, would be an Enterprise Resource Planning (ERP)-system. But for reasons of personal, expenses and time (especially considering the small size of the involved companies), the implementation of such an ERP-system is not applicable.

Since each of the partner companies internally already uses general financial business software to process customer requests, offers and orders (including the calculation and creation of invoices), there was no need to search for these kind of software tools.

Shortly summarised, the main problem areas that have been identified for the Virtual Enterprise are:

- lack of documentation (intra- and inter-organisational),

- order tracking (internal and towards customers),

- project coordination and management,

- information retrieval (for materials, tolerances, measures etc.) and

- capacity and resource planning

In order to achieve a benefit for the VE as a whole, the decision was to select and implement a project management solution to primarily support the company spanning processes.

The introduced approach for improving the "Rapid Development VE« (requirements engineering, workflow optimisation and IT-selection) is mainly based on the results and experiences of the still ongoing EU-project e-MMEDIATE (www.eu-emmediate.com). The objective of this project is to supply the European Community with Best Practices, which shall eventually encourage other interested SMEs on the way of successfully building and maintaining a Virtual Enterprise.

\section{FUTURE ASPECTS}

In the context of Virtual Enterprising, with far-reaching aspects for the future, the companies Cirp, Kurz and Leotech are currently also participating in the EU-project ARICON (www.aricon.org). Within this project, available best practices of VEs are analysed in order to identify qualitative and quantitative success criteria, which form the essential base for the development of capability assessment models for evaluating a company's individual performance to enter into inter-organisational cooperations from business, organisational, legal, technical, ICT and human points of view.

During the project a methodology and tool for the standardisation of guidelines will be developed. Moreover the editing of the first European "Handbook for Virtual Enterprising" will be initiated, which at the long term shall become the key reference for interested companies. This handbook covers all steps from joining/ building a VE, maintaining it up to leaving the VE. Using a metaphor, while in the old economy German engineers used the "Dubbel" (the reference book for 
engineering facts in general), future European managers will use the "ARICON" as the reference for Virtual Enterprising.

\section{CONCLUSION}

Even though there are various examples of very successful Virtual Enterprises, it is obvious that there is still a lot of need for improvement on the way of getting close to something like the perfect VE. Especially the inherited problems that arise from existing cultural, organisational and social traditions between cooperating partners from different countries leave much room for future research. Another issue for consideration is also the need to compensate the differences between VE-partners that greatly vary in their size, like the number of employees or complexity of products.

\section{Acknowledgements}

The work presented in this paper was made possible by the highly appreciated support through the European Commission in funding the research projects:

- e-MMEDIATE (IST-2001-35104)

Electronic Managing of product Manufacturing, Engineering, Design and Investment Applying information Technology for SMEs

- ARICON (GRD1-2001-41865)

Standardised Assessment of Readiness and Interoperability for

Cooperation in New Product Development in Virtual Organisations

The authors would especially like to thank the partner companies Cirp Informationssysteme und Rapid Prototyping GmbH (Cirp), Josef Hofmann Modellbau + Vorrichtungsbau (Hofmann), Modellbau Ralph Kurz (Kurz), Leotech Rapid Prototyping und Werkzeugbau GmbH (Leotech), Profactor GmbH (PROFACTOR), Consiglio Nazionale delle Ricerche (CNR-ITIA), Innovation Network Austria Dienstleistungs-GmbH (INNA), SchlumbergerSema sae (SEMA) and their representatives for their valuable contributions. 